\title{
EXPERIENCIAS Y VIVENCIAS DE CONDICIONES LABORALES EN MÚSICOS DE UNA ORQUESTA SINFÓNICA DEL ECUADOR; UN ENFOQUE FENOMENOLÓGICO
}

\author{
Janeth Fernanda Jiménez Rey ${ }^{1}$, Mónica Isabel Contreras Estrada ${ }^{2}$ y Miguel Alfonso Mercado \\ Ramírez ${ }^{2}$ \\ ${ }^{1,2 \text { y } 3}$ Universidad de Guadalajara, México. ${ }^{1}$ janethfernandajr@gmail.com, ${ }^{2}$ moce10@yahoo.com.mx \\ 3ortomiguel@yahoo.com.mx
}

\begin{abstract}
Resumen. En nuestro estudio se conocerá las experiencias y vivencias de las condiciones laborales, en los músicos de la Orquesta Sinfónica del Ecuador, por su connotación cultural nacional e internacional, quienes se encuentran inmersos, en factores de riesgo. Se considera imperiosa la necesidad de impulsar el diseño e implementación de políticas públicas. Objetivos es comprender las condiciones laborales en los músicos de la orquesta sinfónica. Metodología de estudio es cualitativo con diseño fenomenológico. Se toma en una perspectiva que va desde lo etic a lo emic. Resultados, el análisis de datos cualitativos se obtuvo mediante tres fases; descripción, reducción o tematización e interpretación. Conclusiones Las relaciones vividas en la familia, se describe una profesión en herencia y cultura, conviven por años, en el tiempo existen alegrías, emociones, competencia, ego, fama, y sobre todo sacrificio, en espacio vivido en ensayos y conciertos, dedicado largas horas a su profesión, admás desde su infancia han sacrificado muchas situaciones a su edad, Dentro de los interpretado en los hallazgos de condiciones laborales, se encontró de forma predominante riesgo ergonómico por presencia de lesiones por trastornos neuromusculares a repetición, llevando a licencias o ausencias de trabajo, por periodos renuentes.
\end{abstract}

Palabras clave: Músicos; Orquesta Sinfónica; Condiciones Laborales; Fenomenología.

\section{EXPERIENCES AND EXPERIENCES OF WORKING CONDITIONS IN MUSICIANS OF AN ECUADOR SYMPHONY ORCHESTRA, A PHENOMENOLOGICAL APPROACH}

\begin{abstract}
In our study, the experiences and experiences of working conditions will be known in the musicians of the Ecuador Symphony Orchestra, due to their national and international cultural connotation, who are immersed in risk factors. The need to promote the design and implementation of public policies is considered imperative. Objectives is to understand the working conditions in the musicians of the symphony orchestra. Study methodology is qualitative with a phenomenological design. It is taken in a perspective that goes from the etic to the emic. Results, the analysis of qualitative data was obtained through three phases; description, reduction or thematization and interpretation. Conclusions The relationships lived in the family, a profession in inheritance and culture is described, they coexist for years, in time there are joys, emotions, competition, ego, fame, and above all sacrifice, in space lived in rehearsals and concerts, dedicated long hours to their profession, and since childhood they have sacrificed many situations at their age. Among those interpreted in the findings of working conditions, ergonomic risk was predominantly found due to the presence of repetitive neuromuscular disorders injuries, leading to licenses or absences of work, for reluctant periods.
\end{abstract}

Keywords: Musicians; Symphony Orchestra; Working Conditions; Phenomenology. 


\section{INTRODUCCIÓN}

La siguiente investigación se aborda en base a las condiciones laborales de los músicos de Orquesta Sinfónica de Loja, las cuales son inherentes a su perfil profesional; por lo tanto, su actividad musical es de relevancia e impacto sociológico.

Se considera imperiosa la necesidad de impulsar el diseño de políticas públicas, ya que en nuestro país se evidencia una falta de implementación de sistemas en seguridad y salud para esta población, a pesar que están expuestos a múltiples factores de riesgo. El problema será expresado de forma emic y etic, el ser músico y pertenecer a la Orquesta Sinfónica de la ciudad de Loja y lo que implica el puesto de trabajo al que pertenecen. Por ellos formulamos la pregunta para conocer cuál son las experiencias y vivencias de las condiciones laborales en los músicos de Loja-Ecuador. Nos condujo a obtener los elementos básicos del lenguaje musical, que permite el acercamiento al discurso de ser músico y pertenecer a la organización.

El tipo de trabajo que ellos realizan, es contemplado como uno de los puestos laborales mejor remunerados para los músicos profesionales del país, y además solo existe una Orquesta Sinfónica por región. Pero en qué se traduce realizar estas actividades musicales como ensayos y conciertos, cuál es el costo beneficio tanto en su actuar individual, como colectivo y si con ella, existe una actitud abierta, desinteresada y respetuosa.

El análisis de riesgos planteado no sería bien entendido si no es explicado el marco organizativo en donde se desarrollan y producen las Orquestas sinfónicas, las características que presentan son causa, y se estima por ende que solución, se daría a los problemas de salud que padecen los músicos, por cuanto las características de la tarea son fijas ya que los instrumentos se tocan de una determinada forma sin margen de cambio (Millán ,2012).

La Orquesta sinfónica de la provincia de Loja (OSL), es nuestro objeto de estudio, se ubica en el sur de Ecuador, en la zona geográfica conocida como región interandina o sierra, situada en su capital administrativa, ciudad de Loja habitada por 453.966 personas. La música representa en la ciudad de Loja una de las experiencias artísticas más importantes que identifica su cultura. Destacados músicos y compositores han resaltado a nivel nacional e internacional en el campo cultural de esta ciudad. Hasta el día de hoy la música lojana significa un gran aporte para la cultura nacional. 
A Loja se la conoce en Ecuador como "la capital musical del país", por el número de personas dedicadas al estudio formal de la música en el conservatorio de música, Salvador Bustamante Celi, compositor que a inicios del siglo pasado rebasó las fronteras nacionales gracias a la extraordinaria calidad de sus composiciones sinfónicas.

Por lo tanto, este estudio aborda la importancia de ser músico y pertenecer a una organización representativa de la región, como son las Orquestas sinfónicas, por tanto, es necesario que los músicos tengan buenos sistemas de seguridad e higiene en el trabajo, mejorando su bienestar individual y colectivo. Nuestro aporte se basa en mejorar las políticas públicas de nuestros músicos y que no sean olvidados, es un trabajo que conlleva a la exposición factores de riesgo de manera reiterada, que a lo largo del tiempo perjudicará es su calidad musical y desenvolvimiento escénico.

\section{METODOLOGÍA}

La metodología de esta investigación abarca el diseño cualitativo de las ciencias sociales y del comportamiento, para referirse a dos tipos diferentes de descripción relacionadas con la conducta y la interpretación de los agentes involucrados, en este caso, los músicos de la orquesta sinfónica. Se utiliza una serie de herramientas metodológicas, es decir un mapa de actores sociales, códigos, categorías, el tipo de estudio para esta investigación es fenomenológico, con delimitación espacio temporal, los sujetos participantes, en este caso los músicos de la Orquesta Sinfónica de Loja, utilizando un muestreo por conveniencia. Nuestro proceso del acercamiento a nuestros músicos para sumergirnos en el planteamiento del problema, va de lo etic a lo emic, según Kenneth Lee Pike (1912-2000), mencionaba un nuevo rumbo hermenéutico para encaminar el programa de la antropología, para una vision "interna" versus una "externa" de un mundo social particular (Schaffhauser,2010).

\subsection{Diseño Cualitativo}

Es un estudio se centra en un enfoque cualitativo, fenomenológico que estudia la vida cotidiana, desde las angustias, preocupaciones, en una intersubjetividad con sus semejantes, constituyendo la existencia social. Por ello esta teoría tiene el espacio y tiempo privilegiados en la vida presente y relación cara a cara.

Se justifica la elección en base en el planteamiento del problema y los objetivos debido a la necesidad de explorar el fenómeno en su contexto natural y obtener un entendimiento 
subjetivo (motivaciones, valores, sentimientos y pensamientos). Esto con el fin de conseguir un sentido o una interpretación a partir de los significados que las personas conceden al proceso vivenciado.

\subsection{Delimitación espacio-temporal}

Nuestro estudio está ubicado la ciudad de Loja, parroquia El Valle, al norte de la ciudad, su organización es gubernamental y posee su propio teatro llamado Benjamín Carrión Mora.

\subsection{Sujetos participantes}

Son los músicos de la Orquesta Sinfónica de la ciudad de Loja, quienes ejecutan instrumentos de viento, percusión, cuerdas.

Los participantes serán por conveniencia para nuestra población de músicos que pertenecen a la orquesta sinfónica de Loja, ya que no se conoce cuántos sujetos tienen el fenómeno que nos interesa.

La conveniencia de la estructura de los sujetos participantes y así la conveniencia de nuestra estrategia elegida para lograr obtener ambos, se puede obtener de acuerdo a las preguntas de investigación, por tanto, la pregunta sería, por un lado, qué casos serán necesarios para responder a las preguntas de estudio o cuántos, y por otra parte, la conveniencia de la muestra seleccionada se puede evaluar en función del grado de capacidad de generalización por el que se desea obtener (Flick, 2004). Según Patton (2002) señala que los tipos de diseños "no se parte de un número determinado de antemano" por tanto, la incorporación de las unidades de observación se hace en forma iterativa, de acuerdo con la información que vaya surgiendo en el trabajo de campo, es así que el reto es simplificar las notas de campo y las transcripciones textuales, por ello es necesario el proceso de decodificación que permita desarrollar una clasificación manejable o sistema de códigos (Álvarez,2017).

\subsection{Criterios de selección}

Criterios de inclusión, se contemplan a los músicos que sean profesionales, que tengan nombramiento y trabajen 6 horas por semana en adelante, y que tengan mínimo 10 años en la orquesta sinfónica de Loja. 
Criterios de exclusión, que sean músicos invitados temporales, es decir para conciertos esporádicos, o que estén reemplazando alguno de los músicos de forma eventual, y que sean contratados mediante servicios profesionales.

Criterios de eliminación, se tienen en cuenta los agentes quienes no acepten contestar a las entrevistas, participar en el estudio o dejarlo a medias.

\section{PROCEDIMIENTO DE RECOLECCIÓN}

\subsection{Entrevistas en profundidad}

Siguen el modelo de una conversación entre iguales, y no de un intercambio formal de preguntas y respuestas, no se asemeja a un robot recolector de datos, el propio investigador es el instrumento de la investigación, y no lo es un protocolo de entrevista, el rol implica no sólo obtener respuestas, sino también aprender qué preguntas hacer y cómo hacerlas (Taylor \& Bogdan, 2008). En nuestra investigación se realizó cinco entrevistas en profundidad, logrando entender la realidad social a través de la fenomenología, mediante videos y audios de voz, para conseguir la información anhelada y luego poder transcribirla a conformidad.

\subsection{Notas de observación}

A través de las notas se registra todo aquello que se observa en una entrevista, se determinan mínimos detalles, que a simple vista no los podemos determinar, de tal forma que se recogen sensaciones, expresiones, gesticulaciones, que en la psicología pueden ser interpretadas como un lenguaje universal. A diferencia de la observación vulgar y cotidiana, la observación directa se caracteriza por ser científica y comienza con la selección de un escenario en relación con un determinado tema de investigación (Serrano 2004).

\section{ANÁLISIS}

Las notas de campo constituyen lo apropiado para la fusión entre el estudio de campo empírico y el procesamiento teórico no solo por la secuencia, sino por las dudas que se puedan ir presentando en el camino del investigador. Las dudas y acontecimientos que se presenten en un momento dado para el etnógrafo son decisivas a la hora de inscribir, transcribir y describir el campo (Arenas, 2001). Por este motivo, en nuestro estudio se realizó el análisis mediante el hallazgo por medio de categorías y subcategorias, en donde se describe mediante la entrevista a profundidad el estado fenomenológico social dentro de 
la empresa a evaluar, consiguiendo tener un criterio elocuente de lo empírico a lo científico, luego de los resultados obtenidos al final. Para ello se realizará primero en nuestra investigación la recopilación de ideas y principios detallados por parte del sujeto, con lo cual se emitirá interpretaciones contextualizadas sin admitir especulaciones y propender a la ecuanimidad del estudio a abordar. La explicación está de acuerdo a los cuatro existenciales básicos del enfoque fenomenológico (Van Mannen 1999).

Relaciones humanas vividas (relacionalidad o comunalidad). De acuerdo a la premisa de Merleau-Ponty (1952) que el mundo no es lo que yo pienso sino como lo vivo (Chaverra y Uribe, 2007), se contempla como vive y se relaciona el participante con las personas que trabaja; Espacio vivido (espacialidad). Se considerarán las experiencias, vivencias, el uso de creencias y costumbres en su lugar de trabajo. La información que permitirá visualizar las experiencias representativas positivas y negativas de los trabajadores que se dan en su ámbito laboral. Tiempo vivido (temporalidad). Incluye los aspectos en relación al manejo de los tiempos observados a través de los verbos (pasado, presente, futuro) que se apega con el espacio vivido. Se transparenta la intersección de mi experiencia con otras, todo hombre es temporal en virtud de una necesidad interior en nosotros (Merleau-Ponty, 1985).

Cuerpo vivido (corporeidad). Se refiere a las vivencias y el uso del cuerpo que tiene lugar a partir del momento en que ocurre la ruptura vital (Merleau-Ponty, 1975). Para las narraciones se considerarán las manifestaciones y percepciones en la salud de los participantes, como se visualiza en la tabla 1.

Tabla 1 Categorías y códigos de los cuatro componentes fenomenológicos de los músicos de la orquesta de Loja.

\begin{tabular}{|l|c|l|}
\hline COMPONENTES & CATEGORÍAS & CÓDIGOS \\
\hline \multirow{3}{*}{$\begin{array}{l}\text { Relaciones } \\
\text { humanas vividas }\end{array}$} & En la familia & $\begin{array}{l}\text { Herencia; Apoyo, Padres; Pareja; Hijos; Incentivar; Motivación; } \\
\text { Ocio; Rechazo, Vicios. }\end{array}$ \\
\cline { 2 - 3 } & En el trabajo & $\begin{array}{l}\text { Equipo; Amigos; Unión; Responsabilidad; Tareas; Repasos; } \\
\text { Sincronía, Apoyo; Satisfacción por el trabajo; estrés; falta de } \\
\text { reemplazos; Falta de apoyo por autoridades; competencia, } \\
\text { perfección, ascensos, fata de audiciones. }\end{array}$ \\
\hline \multirow{3}{*}{ Espacio vivido } & Con el público & Alegría; Satisfacción; Agradecimiento; Concurrencia; Apoyo. \\
\cline { 2 - 3 } & Ensayos & Todo el tiempo; Teatro; Casa; Fines de semana. \\
\hline \multirow{2}{*}{ Tiempo vivido } & Concierto & $\begin{array}{l}\text { Luces; Apremiante; Escenario; Aplausos; Composición; Arte; } \\
\text { Cultura; Interacción. }\end{array}$ \\
\hline \multirow{3}{*}{ Cuerpo Vivido } & Infancia & Hermosa; Sacrificada; diferente; dos tareas a la vez. \\
\cline { 2 - 3 } & $\begin{array}{c}\text { Síntomas } \\
\text { corporales }\end{array}$ & $\begin{array}{l}\text { Lesiones; Fatiga; Adormecimiento; Rigidez; Cansancio; Pérdida de } \\
\text { movimiento. }\end{array}$ \\
\cline { 2 - 3 } & $\begin{array}{c}\text { Síntomas } \\
\text { emocionales }\end{array}$ & Autoestima; Ego; Ansiedad; Depresión; Frustración. \\
\hline
\end{tabular}

Fuente: Elaboración propia a partir de la descripción y reducción de las entrevistas en profundidad 


\section{RESULTADOS}

\subsection{Hallazgos}

Las entrevistas en profundidad realizadas en nuestro estudio, se basan en el diseño previo de un guían. Se da inicio con la recolección de datos sociodemográficos para luego receptar el relato del músico, en donde menciona con sus palabras que piensa y percibe de los siguientes ejes temáticos; como el significado de ser músico, su vida cotidiana profesional, las condiciones laborales plasmadas en base a sus ventajas y desventajas, de su antigüedad dentro de la organización, además su estabilidad laboral, su salario, si poseen equipos de protección personal para músicos, que piensan de sus condiciones laborales, si tienen o no chequeos médicos ocupacionales y si han sufrido lesiones a causa de su profesión y de esta forma conseguimos obtener las categorías y subcategorías que darán sentido a nuestro estudio. A continuación, a través de la fenomenología obtendremos el análisis mediante fases, como se detalla en la tabla 2.

Primera fase, de la descripción presentando el fenómeno de las experiencias y vivencias tal como aparece en la conciencia del participante músico y descripción de la información según el esquema de Patton (2002), iniciamos con el cambio de su nombre, procurando su confidencialidad, por nombres alusivos a cantautores de música clásica.

Entrevista 1: Mozart

Entrevista 2: Vivaldi

Entrevista 3: Francesca Caccini

Entrevista 4: Elizabeth Jaquet

Entrevista 5: Beethoven

Segunda Fase, mediante la reducción fenomenológica de las vivencias y experiencias de las condiciones laborales de los músicos de la orquesta sinfónica de Loja se inició con la lectura comprensiva de los textos de las entrevistas y la codificación de los hallazgos, los cuales se fueron descartando a medida que se hacía una nueva lectura.

Los códigos encontrados se agruparon en categorías y en familias de códigos. Se procedió a la definición de cada uno de ellos. Se realizó una representación gráfica de los códigos de forma manual y posteriormente con la ayuda de software para el análisis cualitativo. 


\section{Tercera Fase Interpretación de las vivencias y experiencias de las condiciones} labores de los músicos de la orquesta sinfónica de Loja.

Tabla 2 Descripción general de los hallazgos según esquema de Patton(2002)

\begin{tabular}{|c|c|}
\hline Descripción & Hallazgos generales \\
\hline \multirow{5}{*}{$\begin{array}{r}\text { Experiencia y } \\
\text { comportamiento }\end{array}$} & Mozart: “...directores, obligaciones, competencia, lucha, nos quieren quitar las plazas...” \\
\hline & $\begin{array}{l}\text { Vivaldi: “...Música, Infancia, trabajo, responsabilidad, imagen, estiramientos, puntualidad, } \\
\text { esforzarnos, bebedores sociales...”. }\end{array}$ \\
\hline & $\begin{array}{l}\text { Francesca Caccini: “...Guiado por el director, talento, enfermedad, me ha cambiado mi } \\
\text { vida, concierto, ensayo, estabilidad laboral, sustento económico...”. }\end{array}$ \\
\hline & Elizabeth Jaquet: “...linda, agotadora, ensayos, conciertos ...”. \\
\hline & $\begin{array}{l}\text { Beethoven: “...Nombramiento definitivo, volvería a ser músico, quería ser músico, oveja } \\
\text { negra de la casa...”. }\end{array}$ \\
\hline \multirow{5}{*}{ Opinión y valor } & Mozart: “...No es difícil porque te gusta, no hay reemplazo, fama, eres conocido...”. \\
\hline & $\begin{array}{l}\text { Vivaldi: “...Forma de vida, Forma de expresión, concebida como no profesional, poco } \\
\text { presupuesto, sustento, riesgos laborales, gubernamental, inversión, no compensa, } \\
\text { propias herramientas, hay que ser realistas...". }\end{array}$ \\
\hline & $\begin{array}{l}\text { Francesca Caccini: “...No depende de nosotros, somos gente pública, tenemos que dar } \\
\text { lo mejor, somos críticos, no hay beneficios...". }\end{array}$ \\
\hline & Elizabeth Jaquet: “...Borrón y cuenta nueva, nivel parejo...”. \\
\hline & $\begin{array}{l}\text { Beethoven: “...Malas conductas asociadas, bohemia, alcohol, fama, ego, la directora } \\
\text { terrible que sea mujer...”. }\end{array}$ \\
\hline \multirow{5}{*}{ Sentimientos } & $\begin{array}{l}\text { Mozart: “...Nos gusta la música, la extraño, sacar lo que tiene uno dentro, manifestarse, } \\
\text { sobrevivir, olvidarse de todo, liberarse, contratar...". }\end{array}$ \\
\hline & $\begin{array}{l}\text { Vivaldi: “...Satisfacción, sentirse identificado, forma de expresión, llena el alma, llena el } \\
\text { espíritu, tranquilizarte, deprimirte, me gusta hacerlo, motivación, falta reconocimiento, } \\
\text { pedacito de vida, competitividad, estancados...". }\end{array}$ \\
\hline & $\begin{array}{l}\text { Francesca Caccini: “...trabajo en equipo, coincidir, alma, sentimiento, depresión, } \\
\text { ansiedad, pastillas para dormir, valora la gente, satisfacción personal...”. }\end{array}$ \\
\hline & Elizabeth Jaquet: “...La música es mía, es un espacio mío, estudiar cada día más...”. \\
\hline & Beethoven: “...Satisfacciones, decepciones...” \\
\hline \multirow{5}{*}{ Conocimientos } & Mozart: “...Estudiar siempre, destrezas..." \\
\hline & $\begin{array}{l}\text { Vivaldi: “...Estudios, graduación, licenciatura musical, arte, profesionalización para ser } \\
\text { músico. Trascendencia, saber interpretar, saber escuchar, conciertos, conservatorio, } \\
\text { desconocimientos de nuestros padres, capacitaciones, patrones, años de experiencia..." }\end{array}$ \\
\hline & $\begin{array}{l}\text { Francesca Caccini: “...Difícil encontrar especialización en nuestro medio, estabilidad } \\
\text { laboral...”. }\end{array}$ \\
\hline & $\begin{array}{l}\text { Elizabeth Jaquet: “...Música con responsabilidad, metas, objetivos, me quedo } \\
\text { estudiando, hasta las } 12 \mathrm{~h} 10 \mathrm{pm} \text { repasando, audiciones..." }\end{array}$ \\
\hline & $\begin{array}{l}\text { Beethoven: "....No puede dejar de tocar, destrezas, asimilamos los gestos, } \\
\text { perfeccionista...". }\end{array}$ \\
\hline \multirow{5}{*}{ Sensoriales } & Mozart: “...Melodía, sonido, subir los bajos...”. \\
\hline & $\begin{array}{l}\text { Vivaldi: "....Instrumento, Ritmo, sonidos, nadie lo puede explicar, pero todos lo pueden } \\
\text { sentir..." }\end{array}$ \\
\hline & Francesca Caccini: “...suene bonito, que el público lo sienta ...”. \\
\hline & Elizabeth Jaquet: “....Orgullo, felicidad, dicha..." \\
\hline & Beethoven: “...ruidos, sonidos, melodías, armonías, afinar, domar. \\
\hline \multirow{5}{*}{ Demográficas } & Mozart: “...Antes había güiras...”. \\
\hline & $\begin{array}{l}\text { Vivaldi: “...Público de la costa, Loja las mismas caras, cien por ciento lojano, orquesta } \\
\text { sinfónica de Loja las mejores puntadas, falta oportunidades...". }\end{array}$ \\
\hline & Francesca Caccini: “...viajes, lugares, colonia de lojanos...”. \\
\hline & Elizabeth Jaquet: “...viajes, güiras...”. \\
\hline & Beethoven: “...Movimiento culturales por Loja...”. \\
\hline
\end{tabular}

Fuente: Elaboración propia a cinco músicos de la Orquesta Sinfónica de Loja. 
En la tabla 3 como se menciona a continuación, se da una interpretación de ser músico de la orquesta sinfónica de Loja y las condiciones laborales a las que se encuentra expuestos, analizadas desde los factores de riesgos ocupacionales como ergonómicos, físicos, químicos, biológico, mecánico y hasta psicosociales, si los hubiera a partir de la descripción y reducción fenomenológica.

Tabla 3 Análisis fenomenológico de condiciones laborales de los músicos de la orquesta sinfónica de Loja.

\begin{tabular}{|c|c|c|}
\hline CATEGORÍAS & SUBCATEGORÍAS & SEGMENTO -MOZART \\
\hline \multirow{16}{*}{$\begin{array}{l}\text { CONDICIONES } \\
\text { LABORALES }\end{array}$} & Ergonómico & "...Duele el brazo, o duele el omóplato..." \\
\hline & Físico & $\begin{array}{l}\text { "...Llegue a decirle a mi esposa ponme un paño frio me duele el oído ..." } \\
\text { "....son obras muy fuertes..." } \\
\text { "...la orquesta tiene su ubicación, nosotros tocamos tras los violonchelos..." } \\
\text { más con el dolor de oído ..." ocurió, ponernos a lado de los cornos, no podía } \\
\text { "....debía haber protestado, creo porque era joven..." }\end{array}$ \\
\hline & Psicosociales & "...líneas melódicas muy sensible, existe llanto..." \\
\hline & SUBCATEGORÍAS & SEGMENTO -VIVALDI \\
\hline & Ergonómico & $\begin{array}{l}\text { "...para evitar lesiones..." } \\
\text { “...hacer estiramientos..." } \\
\text { "...los movimientos son repetitivos" } \\
\text { "...curso de fisioterapia tuve que seguir ..." }\end{array}$ \\
\hline & Físico & $\begin{array}{l}\text { "...clima variable..." } \\
\text { "...lesiones y riesgo laboral" } \\
\text { "....nadie entiende, desde nuestra autoridad, propias autoridades médicas" } \\
\text { ".....una presión sonora de dos o tres horas..." } \\
\text { "...no nos han dado equipos de protección personal..." }\end{array}$ \\
\hline & SUBCATEGORÍAS & SEGMENTO -FRANCESCA CACCINI \\
\hline & Ergonómico & 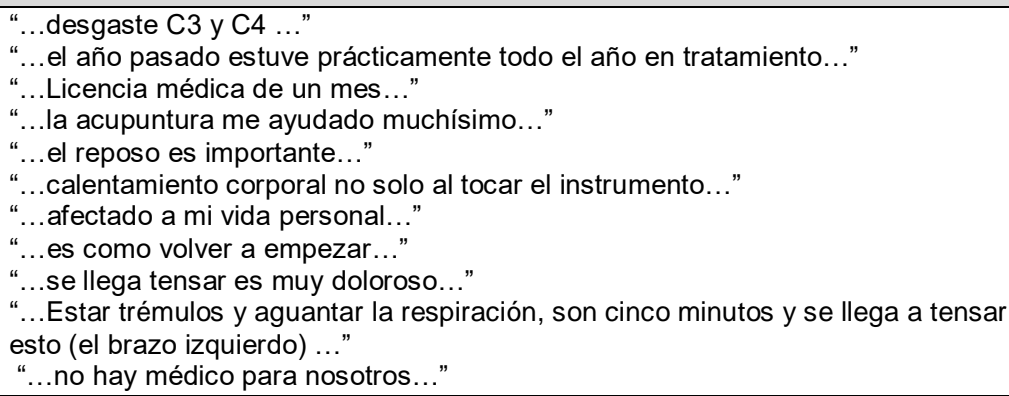 \\
\hline & Físico & “...no dotan de equipos de protección personal, propios para músicos..." \\
\hline & Biológico & "...cuidarnos más nuestro cuerpo..." \\
\hline & Psicosocial & $\begin{array}{l}\text { "....ansiedad..." } \\
\text { "...tuve depresión, ansiedad..." } \\
\text { "...tomaba pastillas para dormir..." }\end{array}$ \\
\hline & SUBCATEGORÍAS & SEGMENTO -BEETHOVEN \\
\hline & Ergonómico & $\begin{array}{l}\text { "...tuve bursitis..." } \\
\text { "...mi músculo se llenó de agua, no inmovilizar, pero sacar el agua y tomar } \\
\text { tratamiento..." } \\
\text { "...licencia un mes ..." }\end{array}$ \\
\hline & Psicosocial & $\begin{array}{l}\text { "...mi ansiedad un tope muy alto..." } \\
\text { "...por mi divorcio, problemas familiares que influyeron ..." } \\
\text { "...pedí ir a la fila de atrás siendo principal ..." }\end{array}$ \\
\hline & SUBCATEGORÍAS & SEGMENTO -ELIZABETH JAQUET \\
\hline & Ergonómico & $\begin{array}{l}\text { "...tengo artritis reumática ..." } \\
\text { "...pienso que es hereditario..." } \\
\text { "...me siento muy adolorida..." }\end{array}$ \\
\hline
\end{tabular}

Fuente: Elaboración propia a partir de la descripción y reducción de las entrevistas en profundidad. 


\section{DISCUSIÓN}

A través de los hallazgos obtenidos en nuestra investigación, se generó la respuesta a la pregunta planteada es nuestro estudio, es decir cuál son las experiencias y vivencias de las condiciones laborales en los músicos de las Orquestas Sinfónicas de Loja. Por tanto, logramos el acercamiento con cada uno de los músicos de la orquesta sinfónica quienes pudieron expresar su percepción, que fue congruente con lo mencionado por Merleau-Ponty (1945), en base a las personas que dirigen su organización o empresa, su mundo y vida cotidiana que se traduce en las decisiones que los músicos puedan llegar a determinar ya sea de forma positiva o negativa en bienestar y seguridad laboral, además ellos son constantes cada día en mejorar su trabajo, porque conocen que existen nuevos músicos y más jóvenes que quisieran pertenecer a la fila del instrumento musical que ello entonan. Los músicos profesionales de este elenco, saben que es su sustento de vida y han invertido una vida entera para pertenecer a la orquesta sinfónica, por tanto, tratan de ser los mejores en su arte. Ellos aman su profesión, lo toman como una distracción no como un trabajo, es su forma de vida, extraen lo que sienten a través de su forma de expresión, pero mencionan que falta un reconocimiento gubernamental, traducido en beneficios en su cuidado y bienestar, para con ello disminuir sus patologías, que puede llegar a influir en la calidad de música.

Shultz (1979), señala que los conocimientos construidos están basados en categorías a partir de la comprensión de la realidad social, traducido en lo vivido en su cotidianidad. Por tanto, para averiguar esa cotidianidad, supimos acercarnos al músico a través del guion plateado, al referir que, en el trabajo, los más importantes es la unión, la amistad que ha surgido durante todo el tiempo de ser músico, en su mayoría una amistad desde la infancia. Refieren los músicos ser como cualquier persona famosa con un escenario y reconocimientos por la comunidad, y país, que muchas de las veces son traducidas en ego y fama y al final depresión y frustración, por no alcanzar su objetivo principal y es tener nuevas audiciones que mejore su salario y gratifique todo el esfuerzo realizado.

Es así, que la interpretación de los hallazgos de las condiciones laborales, expresan factores de riesgos a los cuales se exponen de forma continua, como los ergonómicos por presencia de movimientos repetitivos, problemas posturales, sobreesfuerzo. Además, se preocupan porque no existe intervención en fisioterapia y rehabilitación por parte de la organización, 
incluido los chequeos médicos preventivos especializados para músicos, eso crea incertidumbre en su bienestar y salud.

Los músicos han presentado de forma repetida, dolor extenuante en distintas regiones de su cuerpo, que lleva a depresión y alteración emocional, se preocupan de forma constante por la falta de apoyo emocional de facultativos en el área.

Por tanto, el crear una política pública a nivel gubernamental es meritoria para ellos como músicos profesionales, con el objetivo de mitigar lesiones posteriores y contribuir para generaciones de músicos en un futuro. Las condiciones de trabajo que tienen en la actualidad son las mejores en la parte estructural, de hecho, se sienten agradecidos por haberles proporcionado un teatro de calidad y con los requerimientos técnicos y arquitectónicamente necesarios, sin embargo, piden que se complemente de forma prioritaria, con prevención y promoción de salud de forma especializada en el campo laboral de estudio.

\section{CONCLUSIONES}

Las relaciones vividas en la familia, se describe que es una profesión dada como herencia y cultura. Además, algunos de ellos han referido que luego de algunos años, fue vista como una actividad por la sociedad que contribuía al alcoholismo, e idiosincrasia que no era una profesión, e iba ser mal remunerada. Los amigos son vistos como familias, algunas filas de instrumentos tienen una mejor relación que otra, pero por lo general se llevan de forma adecuada.

Conviven tantos años, que han vivido muchas situaciones que implica alegrías, emociones, competencia, ego, fama, y sobre todo sacrificio. Y con el público siente alegría, apoyo, satisfacción, de forma constante. El espacio vivido, fue determinado por los ensayos y los conciertos.

En este caso los ensayos son de impacto para los músicos, por las horas largas que tienen que dedicar a esta profesión. $Y$ el espacio vivido en el eje de conciertos están acostumbrados a los aplausos, a las luces todo lo que conlleva al escenario y su actuar ante él. Tiempo vivido señala las diferentes circunstancias que se han presentado, desde su infancia, teniendo que sacrificar muchas situaciones a su edad, ya que su objetivo principal 
era cumplir con sus obligaciones extracurriculares, como era asistir a las academias o escuelas de música.

El cuerpo vivido ha llevado a que se produzca cambios corporales y emocionales, es decir los principales cambios corporales son las lesiones musculo esqueléticas presentes, además afectaciones y situaciones de estrés, depresión y ansiedad.

Dentro de los interpretado en los hallazgos de condiciones laborales, se encontró de forma predominante riesgo ergonómico por presencia de lesiones por trastornos neuromusculares a repetición, llevando a licencias o ausencias de trabajo, por periodos largos de tiempo. Mucho de las causas implica en lo que ellos muy bien manifiestan, en falta de cuidados desde; nutricionales, médicos, psicológicos y fisioterapéuticos por la actividad que implica la etiología al respecto, incluida la postura que ellos adoptan. Además, los riesgos físicos generados por el ruido, sobre todo cuando existen cambios de ubicación musical no normados, lo que implica una alta incidencia de trastornos de oído es decir hipoacusias.

La Orquesta Sinfónica, tanto los músicos como los directivos han contribuido de forma constante a nuestro estudio, ellos mantienen la esperanza de ser escuchados, y que las políticas públicas que se puedan implementar a través de estudios como los generados en el presente documento o semejantes, generen cambios que trascienda en su vida cotidiana de ser músicos.

Agradecimientos. A la Orquesta Sinfónica de Loja, por la apertura para realizar nuestra investigación durante estos años.

\section{REFERENCIAS}

Álvarez, I. (2017). Instrumento de recolección de datos y análisis de la información. Universidad Autónoma de México. 1-6

Bech, J. M. (2005). Merleau-Ponty: una aproximación a su pensamiento (Vol. 57). Anthropos Editorial.

Cabrera, I. M. (2013). Influencia de la música en las emociones: una breve revisión. Realista: revista de Ciencias Sociales, Humanas y Artes, 1(2), 34-38.

Carretero, A. (2010). Las enfermedades laborales de los músicos. Revista temas para la educación, (Vol 6), 1-6.

Castillo, J. J., \& Prieto, C. (1990). Condiciones de trabajo: un enfoque renovador de la sociología del trabajo (No. 66). CIS.

Flick, L. y Lederman, NG (2004). Investigación científica y naturaleza de la ciencia. Tendencias y problemas contemporáneos en la educación científica. 
Fuster Guillen, D. E. (2019). Investigación cualitativa: Método fenomenológico hermenéutico. Propósitos y Representaciones, 7(1), 201-229.

Heidegger, M. (1996). Os pensadores. São Paulo: Nova Cultural, 304.

Husserl, E. (2012). La idea de la fenomenología. Herder Editorial.

Martínez-Salgado, C. (2012). El muestreo en investigación cualitativa: principios básicos y algunas controversias. Ciência \& Saúde Coletiva, 17, 613-619.

Mercado, V. (2016). El problema del método en Fenomenología de la percepción de Merleau-Ponty. Revista Ciencia y Cultura, 20(37), 9-42.

Merleau-Ponty, M. (2005). The Cambridge Companion to Merleau-Ponty. Cambridge University Press.

Merleau-Ponty, M., \& Cabanes, J. (1975). Fenomenología de la percepción (p. 475). Barcelona: Península.

Millán, M. J. L. (2012). La organización del trabajo y la estructura de la empresa, elementos clave de los riesgos laborales en las orquestas sinfónicas. Federación de Servicios a la Ciudadanía de CCOO.

Minayo, M.C.S. (2009). La artesanía de la Investigación Cualitativa.Salud Investigación,5,117-120.

Taylor, S. J., \& Bogdan, R. (2008). La entrevista en profundidad. Métodos cuantitativos aplicados, 2, 194.

Pedroso, B., \& Pilatti, L. A. (2010). Revisão literária dos modelos clássicos de avaliação da qualidade de vida no Trabalho: um debate necessário. Qualidade de vida: evolução dos conceitos e práticas no século XXI. Campinas, SP: Ipês.

Serrano, R. S. (2004). La observación participante como escenario y configuración de la diversidad de significados. de Observar, escuchar y comprender sobre la tradición cualitativa en la investigación social, México, FLACSO, El Colegio de México, Miguel Ángel Porrúa, 97-131.

Schütz A. (1967). The phenomenology of the social world. Evanston, IL: Northwestern University Press.

Schaffhauser, P. (2010). La dicotomía emic/etic. Historia de una confusión. Relaciones. Estudios de historia y sociedad, 31(121), 257-269. 